\title{
Expression, regulation and function of phosphofructo-kinase/fructose-biphosphatases (PFKFBs) in glucocorticoid-induced apoptosis of acute lymphoblastic leukemia cells
}

Michela Carlet ${ }^{1,7}$, Kristina Janjetovic ${ }^{1,7}$, Johannes Rainer ${ }^{1,2,7}$, Stefan Schmidt ${ }^{2,3,7}$, Renate Panzer-Grümayer ${ }^{4,7}$, Georg Mann ${ }^{4,7}$, Martina Prelog 5,7 , Bernhard Meister ${ }^{6,7}$, Christian Ploner ${ }^{1,7^{*}}$, Reinhard Kofler ${ }^{1,2,7}$

\begin{abstract}
Background: Glucocorticoids (GCs) cause apoptosis and cell cycle arrest in lymphoid cells and constitute a central component in the therapy of lymphoid malignancies, most notably childhood acute lymphoblastic leukemia (ALL). PFKFB2 (6-phosphofructo-2-kinase/fructose-2,6-biphosphatase-2), a kinase controlling glucose metabolism, was identified by us previously as a GC response gene in expression profiling analyses performed in children with ALL during initial systemic GC mono-therapy. Since deregulation of glucose metabolism has been implicated in apoptosis induction, this gene and its relatives, PFKFB1, 3, and 4, were further analyzed.

Methods: Gene expression analyses of isolated lymphoblasts were performed on Affymetrix HGU133 Plus 2.0 microarrays. GCRMA normalized microarray data were analyzed using R-Bioconductor packages version 2.5. Functional gene analyses of PFKFB2-15A and -15B isoforms were performed by conditional gene over-expression experiments in the GC-sensitive T-ALL model CCRF-CEM.

Results: Expression analyses in additional ALL children, non-leukemic individuals and leukemic cell lines confirmed frequent PFKFB2 induction by GC in most systems sensitive to GC-induced apoptosis, particularly T-ALL cells. The 3 other family members, in contrast, were either absent or only weakly expressed (PFKFB1 and 4) or not induced by GC (PFKFB3). Conditional PFKFB2 over-expression in the CCRF-CEM T-ALL in vitro model revealed that its 2 splice variants (PFKFB2-15A and PFKFB2-15B) had no detectable effect on cell survival. Moreover, neither PFKFB2 splice variant significantly affected sensitivity to, or kinetics of, GC-induced apoptosis.
\end{abstract}

Conclusions: Our data suggest that, at least in the model system investigated, PFKFB2 is not an essential upstream regulator of the anti-leukemic effects of GC.

\section{Background}

Glucocorticoid (GC)-induced apoptosis is a phenomenon of considerable physiologic and therapeutic significance. Physiologically, it has been implicated in the shaping of the immune repertoire and controlling immune responses, and therapeutically it has been exploited in the treatment of lymphoid malignancies, most notably childhood acute lymphoblastic leukemia

\footnotetext{
* Correspondence: Christian.Ploner@i-med.ac.at

'Division Molecular Pathophysiology, Biocenter, Medical University of Innsbruck, Innsbruck, Austria

Full list of author information is available at the end of the article
}

(ALL), where good response to introductory hormone treatment predicts a favourable over-all outcome [1]. GCs mediate most of their effects via their cognate receptor (GR), a ligand-activated transcription factor of the large nuclear transcription factor family. GC-induced apoptosis critically depends on sufficient levels of GR and subsequent alteration in gene expression, but the precise nature of the GC-regulated genes responsible for the anti-leukemic GC effects remains elusive (for reviews see) [1-4].

To further address this issue, we previously exploited a comparative expression profiling strategy using "whole 
genome" microarrays (Affymetrix HGU133 plus 2.0) to delineate the GC response in primary lymphoblasts from children suffering from ALL as well as from several other biological systems of GC-sensitivity and resistance [5]. Only a small number of genes were regulated by $\mathrm{GC}$ in the majority of patients, which might reflect the heterogeneity of the disease. Among these, PFKFB2 (6-phosphofructo-2-kinase/fructose-2,6-biphosphatase-2), a key regulator of glycolysis [6], was one of the most frequently regulated genes. It was induced more than 4-fold in all 3 T-ALL cases as well as in the T-ALL cell line CCRF-CEM. More than 2-fold induction was observed in $8 / 10$ children with precursor B-ALL and $1 / 1$ adult with precursor B-ALL, but not in peripheral blood lymphocytes from 2 healthy donors treated with GC (such cells do not undergo apoptosis in response to GC) [5]. Interestingly, the second GC-sensitive ALL cell line tested in this study, 697/EU-3, and mouse thymocytes failed to regulate PFKFB2. In conclusion, PFKFB2 was induced in many primary ALL cells (particularly T-ALLs), but not in non-malignant lymphoid lineage cells, raising the possibility that this gene might play a functional role in GC-induced apoptosis in malignant lymphoid cells such as ALL.

PFKFB2 is a member of a small gene family encoding 4. PFKFB isoenzymes. PFKFBs regulate formation and degradation of fructose-2,6-biphosphate (F-2,6-P2), a signalling molecule that controls glycolysis by regulating phosphofructokinase-1 (PFK-1) activity $[7,8]$. PFKFBs function as bifunctional enzymes: either they catalyze the synthesis of F-2,6-P2, a potent allosteric effector of PFK-1, via their 6-phosphofructo-2-kinase activity, or they degrade F-2,6-P2 to fructose 6-phosphate by acting as a fructose-2,6-biphosphatase. The kinase to biphosphatase activity ratio $(\mathrm{K}: \mathrm{B})$ is determined by the expression of specific isoforms, post-translational modifications of the enzyme as well as by numerous catabolic metabolites such as $\alpha$-glycerol phosphate, citrate or ATP/GTP levels in the cells [9]. Initially identified in rat hepatocytes [8], 4 mammalian PFKFB isoenzymes have been described in different tissues and developmental stages, i.e., PFKFB1 in liver, skeletal muscles and fetal tissues; PFKFB2 in heart, kidney, pancreas islets and lymphoid tissues; PFKFB3 ubiquitously, particularly in brain; and the testis-specific PFKFB4. All isoenzymes share a highly conserved core structure comprising the kinase and phosphatase activities. The isoenzymes and their isoforms mainly differ in their $\mathrm{N}$ - and C-terminal variable regions, however, all of them are active as homodimers where the kinase domains come together in a head-to-head fashion, whereas the phosphatase domains function as monomers [7].

The human PFKFB2 gene codes for 2 isoforms (15A and $15 \mathrm{~B}$ ) generated by alternative splicing of the terminal exon 15, thus differing in their C-terminal amino acid sequence [6]. Interestingly, in the more frequently investigated PFKFB2-15A isoform, this 54 amino acid region contains two PKA-sensitive phosphorylation sites [10], i.e. Ser466 and Ser483, which have been shown to play an important role in the regulation of kinase- and phosphatase activity. Thus, phosphorylation of Ser466 increased kinase affinity to fructose-6-phosphate (Fru-6-P), whereas dephosphorylation of the same residues entailed increased phosphatase activity [11]. Since the PFKFB2-15B isoform lacks the above kinase-activating phosphorylation sites, it might lack kinase activity, raising the possibility that the 2 isoforms may have opposite functions.

Recently, alterations in glucose metabolism have been implicated in cell death and survival decisions, particularly in the lymphoid lineage [12] and in transformed cells [13]. Thus, lymphocytes depend upon extracellular signals transmitted via surface receptors and so-called survival kinases, like PKB/Akt, to maintain their viability. Part of these survival signals impinge on glucose metabolism, e.g., by increasing the expression of glucose transporters [14], with the net outcome of increased glycolysis and ATP production. Decline in glycolytic activity and ATP/ADP ratios results in integrity loss of mitochondria, the central regulators of metabolism and survival [15], with subsequent Bax-Bak dependent cytochrome-C release and cell death. Similarly, tumor cells depend upon glycolytic flux (Warburg effect) [16,17], which provides them with building blocks required for growth and energy. Hence, interference with "tumor metabolism" has become a promising target for cancer therapy [13].

The crucial role of PFKFBs in controlling glycolysis, along with the fact that one member of this small gene family, $P F K F B 2$, was frequently regulated by GC in malignant lymphoblasts from children suffering from childhood ALL [5], makes this enzyme an interesting candidate for the anti-leukemic effects of GC. To further address this issue, we investigated expression and GC regulation of all 4 known $P F K F B$ family members in the previously published and additional childhood ALL patients and in additional in vitro leukemic systems. We found that only PFKFB2 showed consistent $\mathrm{GC}$-induction, particularly, although not exclusively, in T-ALL systems. To assess a possible functional significance of this regulation, we performed conditional over-expression using the 2 known PFKFB2 splice variants in the T-ALL cell model CCRF-CEM in which both variants are dramatically induced by GC. However, neither splice variant replicated the apoptotic GC effects nor entailed over-expression a consistent modulating effect on GC-induced leukemia apoptosis. 


\section{Methods}

\section{Patients, cell lines and tissue culture}

The ALL children enrolled in this study were admitted to the Department of Pediatrics, Innsbruck Medical University, or the St. Anna Kinderspital in Vienna from September to December 2009, and treated according to the BFM protocol 2000 (for protocol details see: http:// boriskononenko.files.wordpress.com/2009/09/1-protokoll-all-bfm-2000-mit-amendments-version-26-09-07d0bad0bed0bfd0b8d18f1.pdf). To analyze the GC response in non-leukemic peripheral blood lymphocytes, Ficoll-purified peripheral blood mononuclear cells from 2 healthy volunteers and 3 children with epilepsia treated with a single injection of GC were included. The study was approved by the Ethics Committees of the Innsbruck Medical University (EK1-1193-172/35 for ALL children and UN2821 for epileptic children) and the Kinderspital in Vienna, and written informed consent was obtained from the patients and/or parents or custodians. The characteristics of 3 children with T-ALL and 10 with precursor B-ALL have been published previously [5], the remaining ALL children (3 T-ALLs and 15 precursor B-ALLs) showed similar clinical findings [Rainer et al. in preparation]. All 3 patients with epilepsia (E3, a 14 year-old girl; E4, a 6 year-old boy; E5, an 11 year-old boy) had an idiopathic generalized epilepsia without any evidence of metabolic, endocrinological, immunological, hematological or infectious disease. The 6 year old girl (epilepsia diagnosed at 4 years of age) was on Valproinate and Clobazam, the 9 year old girl (epilepsia diagnosed at 8 year of age) on Valproinate and Rufinamide, and E5 (epilepsia diagnosed at 2 years of age) on Valproinate and Sultiam. Because of therapyrefractory epilepsia, the 3 patients received their first GC pulse therapy with dexamethasone (Fortecortin, Merck, Vienna, Austria) $20 \mathrm{mg} / \mathrm{m}^{2}$ administered intravenously over 1 hour. Blood samples were taken at 2, 6 and 24 hours after drug administration.

The T-ALL cell lines CCRF-CEM-C7H2 [18], 6 GC-sensitive and $6 \mathrm{GC}$-resistant derivatives CCRFCEM-C7H2 [19], CEM-C7H2-2C8 [20], a CEM-C7H2 derivative with constitutive expression of the tetracycline-regulated reverse transactivator, rtTA [21], MOLT4 (CRL-1582, ATCC, Rockville, MD), and Jurkat (untransfected and a rat GR-transfected derivative [22]), the precursor B-cell lines 697/EU-3 (ACC 42, DSMZ, Braunschweig, Germany), NALM6 (ACC 128, DSMZ), RS4;11 (ACC 508, DSMZ) and AT-1 [23], and Burkitt's lymphoma Daudi (CCL-213, ATCC) were cultured in RPMI 1640 supplemented with $10 \%$ fetal calf serum and $2 \mathrm{mM} \mathrm{L}$-glutamine at $37^{\circ} \mathrm{C}, 5 \%$ carbon-dioxide and saturated humidity. HEK293T packaging cells (ATCC, Manassas, VA) were cultured in DMEM supplemented as above. The cells were free of mycoplasma infection and their authenticity was verified by DNA fingerprinting, as detailed previously [24]. Doxycycline was dissolved $(100 \mu \mathrm{g} / \mathrm{ml})$ in phosphate-buffered saline and dexamethasone $\left(10^{-4} \mathrm{M}\right)$ in $100 \%$ ethanol. The final ethanolconcentration in the dexamethasone-treated and control cultures was maintained at $0.1 \%$. All above reagents were from Sigma (Vienna, Austria).

\section{Expression profiling}

Our procedure for generating mRNA expression profiles on Affymetrix HGU133 plus 2.0 microarrays has been detailed previously [5]. Briefly, RNA from peripheral blood lymphoblasts of patients prior to, or at 6-8 or 24 hours after initiation of systemic GC monotherapy (following the BFM therapy protocol recommendations), from healthy volunteers or epileptic children 6 hours after GC treatment, and GC-sensitive or GC-resistant cell lines, was converted into labeled target and hybridized to Affymetrix HGU133 plus 2.0 microarrays according to standard protocols. The microarray data from the 31 ALL-patients and 5 non-leukemic individuals were normalized separately from those of the 12 cell lines using GCRMA [25] after positive evaluation of comprehensive raw data quality assessment. The analysis was performed in $\mathrm{R}$ using packages from Bioconductor [26] version 2.5. Annotation of probe sets to genes based on Affymetrix NetAffx annotation database version 22 and location of probe sets relative to the 3' end of the transcripts were manually inspected using the Ensembl genome browser for genes PFKFB1, PFKFB2, $P F K F B 3$ and $P F K F B 4$. Microarray data for the samples of the 13 ALL children and 2 healthy donors were published previously [5] and are available at NCBI's GEO database (series GSE2677 and GSE2842). Microarray data for the 12 cell lines (GSE22152) and 4 additional non-leukemic individuals (GSE22779) have been deposited at GEO.

\section{Apoptosis determinations}

Apoptosis was determined by FACS analysis of propidium iodide (PI)-treated permeabilized cells [27] as previously detailed [28]. Briefly, cells were analyzed with a FACScan cytometer (Becton Dickinson Biosciences, San Jose, CA) in combination with CellQuest Pro software (Becton Dickinson Biosciences) acquiring forward scatter/sideward scatter, FL-2 H (log), and FL-3 H (linear). In FL-2 $\mathrm{H}$, the percentage of nuclei with reduced DNAcontent (SubG1 peak) was assessed.

\section{Immunoblotting}

Our immunoblotting procedure has been detailed recently [29]. Briefly, proteins were extracted from $5 \times 10^{6}$ cells 
in $100 \mu$ l RIPA-buffer, quantified by Bradford analysis, mixed with $40 \mu$ l loading buffer $(4 \times$ SSB, $5 \% \beta$-mercaptoethanol), denatured, fractionated on a $12.5 \%$ SDSPAGE and electroblotted onto nitrocellulose. The membranes were incubated overnight with rabbit polyclonal antibodies against PFKFB2 (N-term, AP8146a, Abgent), PFKFB2 phosphorylated Ser466 (University of Dundee, UK) or mouse monoclonal antibodies against $\alpha$-TUBULIN (DM1A, CalBiochem, Nottingham, UK) as a loading control. Specifically bound antibodies were detected with anti-rabbit, anti-sheep or anti-mouse horseradish-peroxidase-conjugated secondary antibodies (Amersham Pharmacia Biotech, Uppsala, Sweden) and visualized by chemiluminescence (ECL, Amersham) and subsequent exposure to AGFA Curix X-ray films for 1 second to 30 minutes.

\section{Generation of CCRF-CEM derivatives with doxycycline- induced PFKFB2-15A and -15B expression}

The lentiviral conditional expression constructs pHRtetCMV-PFKFB2-15A and -15B (U264 and U265) were generated using the GATEWAY ${ }^{\mathrm{Tm}}$ technology (Invitrogen, Carlsbad, CA). The details of this procedure and the generation of stable clonal cell lines with tetracycline-regulated expression of cDNAs cloned into such constructs has been described previously [30]. In brief, human sequences coding for PFKFB2-15A and $-15 B$ mRNA were PCR-amplified using unique SacI-flanked forward primer 5'-GAGCTCTGTGCTCGACGAGCTCGT-3' and XbaI-flanked isoform specific reverse primers 5'-TCTAGATGGGTCTTCGGCTAGT-3' (for PFKFB2-15A) and 5'-TCTAGAGTAGATCCCAGTCGT-3' (for PFKFB2-15B) and cDNA from CEM-C7H2 cells as template. The purified PCR product was cloned into the SacI-XbaI site of pENTR-MCS-deltaNcoI (U243), thereby generating pENTR207-PFKFB2-15A (U260) and pENTR207-PFKFB2-15B (U263). Constructs were sequence-verified and subsequently recombined into the "destination vector" $\mathrm{pHR}$-tetCMV-Dest-IRES-GFP (U192) to generate pHR-tetCMV-PFKFB2-15A-ires-GFP (U264) and pHR-tetCMV-PFKFB2-15B-ires-GFP (U265), as previously described [30]. The lentiviral plasmids were transfected into $293 \mathrm{~T}$ packaging cells together with pVSV$\mathrm{G}$ and pSPAX (kindly provided by Didier Trono), and the lentivirus-containing supernatants were used to transduce CEM-C7H2-2C8, which constitutively express rtTA [20]. After limiting dilution cloning, three clonal cell lines expressing the PFKFB2-15A isoform, termed CEMPFKFB2-15A\#C3, \#D6 and \#E8, and three expressing PFKFB2-15B named CEM-PFKFB2-15B\#65, \#66 and \#95, were selected for further experiments.

\section{Real time RT-PCR}

For high through-put real time RT-PCR, $50 \mu$ l of diluted cDNA $(2 \mathrm{ng} / \mu \mathrm{l})$ were added to $50 \mu \mathrm{l}$ of TaqMan
Universal MasterMix (Applied Biosystems, Foster City, CA) and introduced into microfluidic cards containing real time RT-PCR mixes for human PFKFB2 (Hs01015408_m1, Applied Biosystems) and TATA boxbinding protein for mRNA normalization (TBP, Hs00427620_m1) according to the manufacturer's guidelines. After equilibration at RT, the channels were filled with $100 \mu \mathrm{l}$ of reaction mix and centrifuged two times for 1 minute at $1000 \mathrm{rpm}$. Thereafter, the cards were sealed, loaded into the HT7900 real time machine (Applied Biosystems), and run with a 2-step PCR thermo-protocol that included an initial $94.5^{\circ} \mathrm{C}$ step for $10 \mathrm{~min}$ followed by 40 cycles of $97^{\circ} \mathrm{C}$ for $15 \mathrm{sec}$ alternating with $60^{\circ} \mathrm{C}$ for 1 min. Fluorescence signal intensities were read during the $60^{\circ} \mathrm{C}$ temperature step. Similarly, but on conventional 96 well plates, mRNA encoding the 2 PFKFB2 splice variants 15A and 15B were quantified (Hs01015410_m1 and Hs01016554_m1, Applied Biosystems, for PFKFB215A and PFKFB2-15B, respectively). Primary real-time PCR data analysis was performed with SDS software version 2.2.1, and further analysis was performed in $\mathrm{R}$ (version 2.8). Data from 3 technically replicated measurements were averaged and normalized to the internal TBP control. Log2 fold change values ( $M$ values) were calculated for 3 biological replicates by comparing normalized real-time PCR data from GC-treated samples against data from the corresponding control samples. $M$ values were averaged for the 3 biological replicates and p-values were calculated (Student's t-test) to test against the null hypothesis of no differential expression (mean $\mathrm{M}=0$ ).

\section{Results}

Expression and regulation of PFKFB1, 3 and 4 isoenzymes in lymphocytes and malignant lymphoblasts

In a previous study, we showed that PFKFB2 is regulated by GC in 11/13 children suffering from T-ALL and preB-ALL [5]. To elucidate whether GC exclusively regulated $P F K F B 2$ in lymphoid cells, or whether other PFKFB-isoenzymes are susceptible to GC-treatment as well, we re-analyzed this data using the more robust GCRMA normalization and included 18 additional ALL patients undergoing GC therapy as well as 3 non-leukemic children who received GC as part of their epilepsia treatment, and 6 GC-sensitive and 6 GC-resistant derivatives of the CEM-C7H2 T-ALL cell line. Additional file 1, Table S3 summarizes mean expression levels (mE-value) and mean GC regulation (mM-value) of the 3 isoforms, i.e. PFKFB1, PFKFB3 and PFKFB4, in these samples, organized in the following groups: T-ALLs, precursor B-ALLs [as one group and as 3 separate groups based on molecular defects: hyperdiploid, ETV6/ RUNX1 (previously TEL/AML1) translocation, and a heterogeneous group termed "others"], non-leukemic 
donors and GC-sensitive and -resistant CCRF-CEM derivates. Data for individual patients and cell lines are depicted in Additional file 1, Tables S1 and S2. PFKFB1 and PFKFB 4 isoenzymes were neither detectably expressed in any of the investigated systems nor regulated by GC. The brain/placenta- and tumor-specific PFKFB3 isoenzyme showed expression levels comparable to those of PFKFB2 (for comparisons see Table 1), however, in contrast to $P F K F B 2$, its expression was not upregulated by GC. Interestingly, expression of PFKFB3 was also observed in peripheral blood lymphocytes from non-leukemic donors, indicating that this isoenzyme is not restricted to placenta and brain or malignant tissues. In conclusion, the PFKFB 1 and 4 isoenzymes were neither expressed nor regulated by GC and, hence, might not play a role in the anti-leukemic GC effects. PFKFB3 is expressed, but shows no significant GC-regulation, excluding it from the list of likely candidate genes.

\section{Expression and regulation of PFKFB2-15A and $-15 B$ in lymphocytes and malignant lymphoblasts}

Next, we analyzed expression and GC regulation of the 2 splice variants of PFKFB2 in the above biological systems. As summarized in Table 1, basal expression of both PFKFB2 isoforms was variable: almost all T-ALLs (known to show particularly strong response to GC) and ETV6/RUNX1 positive ALLs (which have a good prognosis) showed intermediate, all other subgroups, no or very low expression. In all groups with detectable expression of $P F K F B 2$, mRNA levels (as measured by signal intensity) of $P F K F B 2-15 A$ were $\sim 2$-fold higher than those of PFKFB2-15B. Concerning regulation, T-ALL patients showed upregulation of both splice variants at both time points, with mean $M$ values between 1.7 and 2.7. This was also true for precursor B-ALL patients, although the extent of regulation was considerably less (mean $\mathrm{M}$ values between 0.6 and 1.1).

On an individual basis (Additional file 1, Table S1), the T-ALL group appeared quite homogeneous, with 5/6 T-ALL children showing induction (as defined by an M-value $>1$, i.e., $>2$-fold) after 6 and/or 24 hours of GCtreatment. In hyperdiploid precursor B-ALL children, isoform PFKFB2-15A was upregulated in 5/7 cases, with one child presenting a tendency for downregulation (Mvalue $=-0.6$ ), while $P F K F B 2-15 B$ induction was present only in 1 of the 7 children after 6 hours. The subgroup of children with the ETV6/RUNX1 translocation showed induction of PFKFB2-15A in 4/10 children, 2 showed a slight downregulation, and 4 lacked $P F K F B 2-15 A$ regulation. A more homogeneous situation was observed for the PFKFB2-15B isoform, where induction was detected in $8 / 10$ children, with the remaining 2 showing a tendency to downregulate $P F K F B 2-15 B$ after the first time point. The last subgroup [low hyperdiploid, $E 2 A / P B X 1$ translocation, $\mathrm{t}(8 ; 4)$ and no chromosomal abnormalities] showed upregulated $P F K F B 2-15 A$ in $7 / 8$ cases and PFKFB2-15B in $4 / 8$, and no regulation in the others. In contrast to the ALL samples where PFKFB2 induction was a frequent event, this was seen only once and only for PFKFB2-15A in the non-leukemic donors. Concerning the in vitro cell lines systems, all $6 \mathrm{GC}$-sensitive CEM-C7H2 subclones showed clear induction of PFKFB2-15A and $-15 B$, with $M$ values ranging from 1.6 to $4.0(15 \mathrm{~A})$ and 1.1 to $2.3(15 \mathrm{~B})$. The $6 \mathrm{R}$-lines resulted in a remarkably heterogeneous picture, with $\mathrm{M}$-values ranging from 0.0 to 5.1 (15A) and 0.5 to 3.5 (15B). Thus, despite the complexity of the expression profiling data, PFKFB2 induction appeared to be a frequent feature in the GC response of T-lineage ALL and also occurred in precursor B-ALL, albeit with lower frequency. Moreover, PFKFB2 induction was absent or

Table 1 Expression and regulation of PFKFB2 in different lymphoid systems ${ }^{1}$

\begin{tabular}{|c|c|c|c|c|c|c|}
\hline \multirow[b]{2}{*}{ samples } & \multicolumn{2}{|c|}{$\begin{array}{l}\text { Expression } \\
\text { (mE-value) }\end{array}$} & \multicolumn{2}{|c|}{$\begin{array}{l}\text { Regulation } \\
\text { (mM-value) }\end{array}$} & \multicolumn{2}{|c|}{$\begin{array}{l}\text { Regulation } \\
\text { (mM-value) }\end{array}$} \\
\hline & $15 A$ & $15 B$ & $15 A 6 / 8 \mathrm{~h}$ & $15 \mathrm{~A} 24 \mathrm{~h}$ & $15 B 6 / 8 \mathrm{~h}$ & 15B $24 \mathrm{~h}$ \\
\hline T-ALL (6) & $6.3 \pm 1.5$ & $4.0 \pm 1.9$ & $1.7 \pm 1.5$ & $2.4 \pm 1.4$ & $1.9 \pm 2.3$ & $2.7 \pm 2.3$ \\
\hline Precursor B-ALL (25) & $4.6 \pm 2.7$ & $3.0 \pm 1.7$ & $0.7 \pm 0.8$ & $1.1 \pm 1.2$ & $0.6 \pm 0.9$ & $0.9 \pm 1.1$ \\
\hline - hyperdiploid (7) & $2.1 \pm 0.7$ & $1.5 \pm 0.2$ & $0.7 \pm 0.6$ & $0.9 \pm 1.1$ & $0.3 \pm 0.4$ & $0.3 \pm 0.3$ \\
\hline - EVT6/RUNX1 (10) & $6.7 \pm 1.7$ & $4.4 \pm 1.5$ & $0.5 \pm 1.1$ & $0.8 \pm 1.5$ & $0.8 \pm 1.1$ & $1.1 \pm 1.2$ \\
\hline - others (8) & $4.2 \pm 2.7$ & $2.4 \pm 1.3$ & $0.9 \pm 0.8$ & $1.5 \pm 0.8$ & $0.6 \pm 0.8$ & $1.3 \pm 1.2$ \\
\hline Non-leukemic donors $\left(6^{*}\right)$ & $3.4 \pm 0.2$ & $2.0 \pm 0.4$ & $0.5 \pm 0.7$ & ND & $0.2 \pm 0.3$ & ND \\
\hline CEM-C7H2-S-lines (6) & $2.3 \pm 0.1$ & $1.8 \pm 0.1$ & $3.1 \pm 0.9$ & ND & $1.8 \pm 0.4$ & ND \\
\hline CEM-C7H2-R-lines (6) & $2.7 \pm 0.3$ & $1.8 \pm 0.1$ & $2.0 \pm 2.0$ & ND & $1.6 \pm 1.3$ & ND \\
\hline
\end{tabular}

${ }^{1}$ Expression and regulation of PFKFB2 in ChALL patients, non-leukemic controls (adult healthy donors and epileptic children) and sensitive (S) and resistant (R) CEM-C7H2 cell lines. Basal mean expression, before GC-treatment, is reported as mE-value \pm SD; mean regulation was assessed after 6 and 24 hours and is expressed as mM-value \pm SD. The total number of cases is reported in brackets next to the subgroup name. Data were assessed by analysis of Affymetrix HGU133 plus 2.0 microarrays. Abbreviation: ND, not determined. 
reduced in systems resistant to $\mathrm{GC}$-induced apoptosis (non-leukemic donors, CEM-C7H2-R-lines).

\section{Expression and regulation of PFKFB2 isoforms in additional leukemic cell line models}

To further address whether PFKFB2 regulation was related to GC-sensitivity and/or preB-ALL and T-ALL origin, we treated 9 leukemia cell lines with $10^{-7} \mathrm{M}$ dexamethasone for 2, 6, 12 and 24 hours in biological triplicates and performed real time RT-PCR using primers covering the exon-3/exon-4 boundary present in both splice variants (Figure 1A). To assess the contribution of the individual splice variants, the 24 hour samples were further analyzed using primers specific for PFKFB2-15A and $-15 B$ (Figure $1 B$ and $1 C$ ). As we previously reported [31], the extent of GC sensitivity in these cell lines was markedly different, i.e., untransfected Jurkat and MOLT4 T-ALL cell lines as well as AT-1 precursor B-ALL and Daudi Burkitt lymphoma cells were resistant to GC-induced apoptosis, while all others were GC-sensitive, although with varying kinetics (Additional file 1, Figure S1). Effects of GC on cell cycle progression were also determined [31] (Additional file 1, Figure S1) and showed that GC-induced apoptosis was frequently preceded or accompanied by an increase of cells in the G1 phase of the cell division cycle, whereas the cell lines resistant to GC-induced apoptosis were also resistant to the GC effects on the cell cycle. The notable exception was AT-1, in which G1 cell cycle arrest was observed in the complete absence of apoptosis. As shown in Figure 1A, PFKFB2 induction was a frequent event in these cell lines starting as soon as 2 hours after initial GC exposure and increasing up to 24 hours. In those instances where PFKFB2 induction was observed, both splice variants appeared to be similarly regulated (Figure 1B). There was no apparent correlation between PFKFB2 induction and GC sensitivity or T/B-lineage origin of the leukemic cell lines. Concerning basal levels, the 2 splice variants showed co-expression across the panel of leukemia cell lines (Figure 1C), with PFKFB2-15A levels slightly exceeding those of $-15 B$, thus resembling the findings on the microarrays. In general, B-lineage cells revealed lower expression levels than $\mathrm{T}$-lineage cells (again resembling the situation in patients), but there was no apparent correlation between basal PFKFB2 levels and GC-sensitivity. For instance, GC-sensitive C7H2 T-ALL cells had similar PFKFB2 levels as GC-resistant MOLT4 T-ALL. The same was true for GC-sensitive Jurkat ${ }^{\mathrm{GR}}$ and their parental GC-resistant Jurkat cell line.

Similar to the situation in patients, GC-dependent induction was more pronounced in T-ALL cells than in B-lineage leukemias, but there was no obvious correlation with GC sensitivity across all samples. In the Jurkat
T-ALL system, however, induction of PFKFB2 correlated with GC sensitivity, as we have similarly seen in the CCRF-CEM system using microarray technology. Although we were unable to definitively prove regulation at the protein level (for reasons explained in Additional file 1), the combined clinical and experimental mRNA data were compatible with the notion that induction of PFKFB2 contributes to GC-induced apoptosis, particularly in T-ALL cells.

\section{Effect of conditional over-expression of PFKFB2 isoforms on cell viability and GC-sensitivity}

To evaluate a possible contribution of increased PFKFB2 levels to the anti-leukemic effects of GC, we generated cell lines conditionally overexpressing either PFKFB2$15 \mathrm{~A}$ or $-15 \mathrm{~B}$ in a doxycycline-dependent manner. Three clones for each isoform (termed CEM-PFKFB2-15A\#C3, \#D6, \#E8 and CEM-PFKFB2-15B\#65, \#66, \#95, respectively) were analyzed for regulation of the transgene by quantitative RT-PCR and immunoblotting (Figure 2 and Additional file 1, Figure S2 and S3). In all instances we obtained doxycycline dose- and time-dependent induction of the PFKFB2 splice variants to levels as high as, or even exceeding, those obtained after GC treatment. Nevertheless, no effect was observed on cell viability, suggesting that induction of either PFKFB2 splice variant is not sufficient to explain apoptosis seen after GC exposure (Figure 3). In addition, the FACS data of PI-stained nuclei strongly suggested that there was no effect on cell cycle progression or GC-induced cell cycle arrest (data not shown). To investigate whether transgenic expression of PFKFB2 resulted in altered GC susceptibility, we treated the above T-ALL cell lines with $10^{-7} \mathrm{M}$ dexamethasone in the presence and absence of doxycycline (i.e., with and without transgenic PFKFB2 induction) and analyzed the extent of apoptosis (as measured by FACS analysis of propidium iodide incorporation) at various time points (Figure 3). Neither PFKFB2-15A nor -15B over-expression significantly changed GC sensitivity in these cells, although both isoforms were clearly detectable by immunoblotting (Figure 3C). Moreover, in the case of PFKFB2-15A where corresponding analyses can be performed, we observed that the activation-specific Ser466 [11] was phosphorylated, suggesting that the transgenic protein was not only sufficiently well expressed, but also active as a kinase. In conclusion, the data show that, at least in the investigated model system, neither PFKFB2-15A nor -15B over-expression mimics the anti-leukemic effects of GC, nor does it alter GC sensitivity.

\section{Discussion}

In this study, we addressed the possible functional significance of GC-dependent PFKFB2 regulation in 

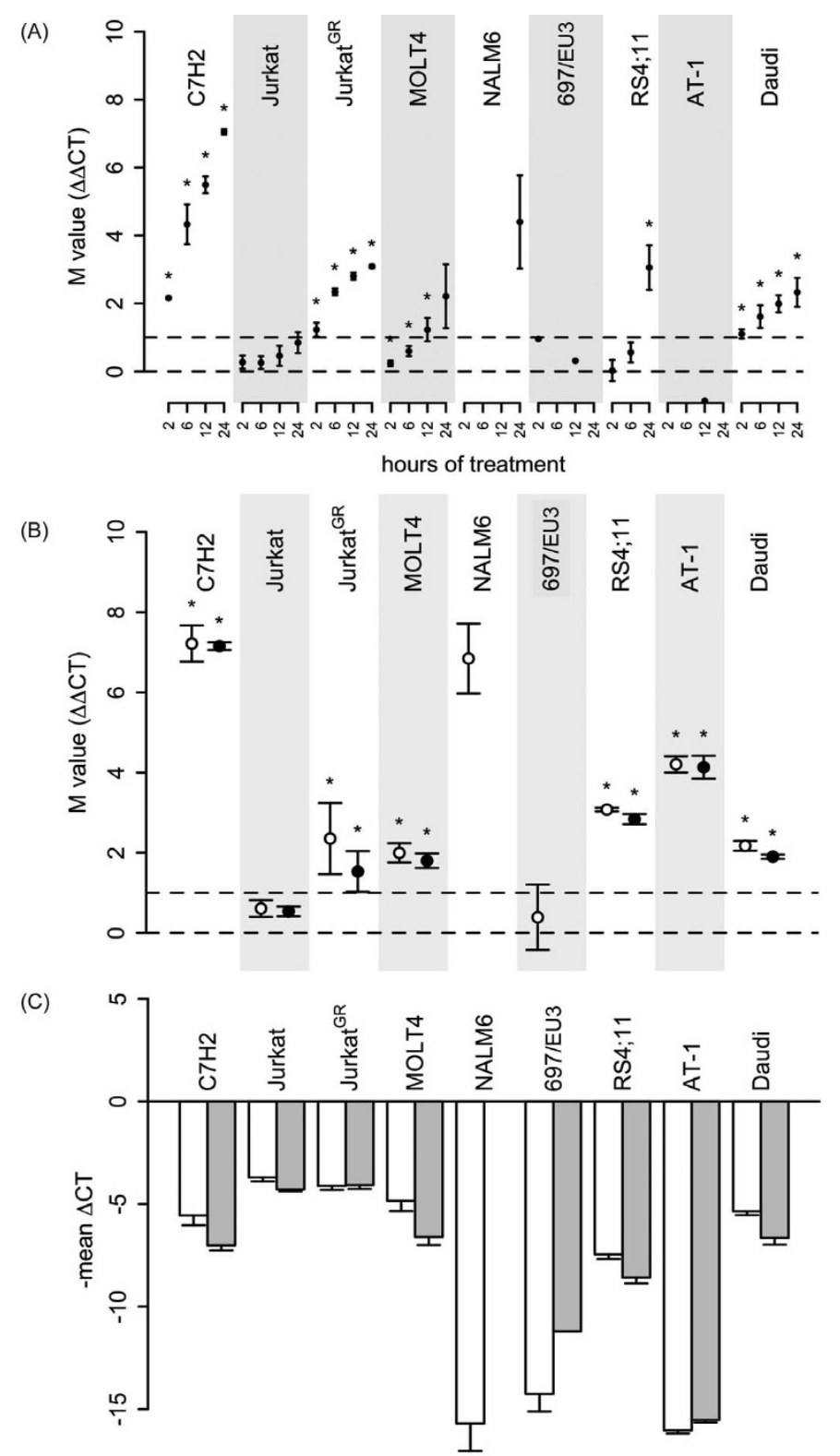

Figure 1 Expression/regulation of PFKFB2 and its splice variants in leukemic cell lines. (A) The cell lines were cultured as indicated in the presence of $10^{-7} \mathrm{M}$ dexamethasone or $0.1 \%$ ethanol as vehicle control, and analyzed for mRNA expression of PFKFB2 (using a primer pair recognizing both splice variants) and TBP as control by quantitative RT-PCR on microfluidic cards. TBP-normalized PFKFB2 expression levels ( $\triangle C_{T}$ ) were used to determine $\mathrm{GC}$ regulation $\left(\triangle \triangle \mathrm{C}_{\mathrm{T}}\right)$ expressed as mean $\mathrm{M}$-values \pm standard deviation. Asterisks indicate $\mathrm{p}$-values of $<0.05$. PFKFB2 was undetectable in NALM6 (at 2,6 and 12 hours) and AT-1 cells prior to, but clearly detectable, after GC treatment. Thus, the extent of regulation $\left(\Delta \Delta C_{T}\right)$ could not be quantified. Asterisks indicate statistically significant changes of expression $(p<0.05)$ as calculated by a paired t-test from 3 replicated values. Regulations were considered biologically significant if the extent of differential expression was larger than 2-fold (indicated by the dashed horizontal line $M=1$ ). (B) Regulation of PFKFB2 variants (open circles for PFKFB2-15A, black circles for PFKFB2-15B) after 24 hour exposure to $10^{-7} \mathrm{M}$ dexamethasone is expressed as mean $\mathrm{M}$-value $(\triangle \triangle C T) \pm S D$. Asterisks indicate $p<0.05$. In NALM6 cells, PFKFB2-15B was induced by GC as well. However, since the expression level in the ethanol-treated control sample was below the limit of detection, it was not possible to derive an M-value. In the 697/EU-3 cells, PFKFB-15B was undetectable after 24 hour GC exposure, which might be explained by the fact that PFKFB2 levels in 697/EU-3 were at the limit of detection of this assay or suggest a weak down-regulation. (C) Basal expression of the PFKFB2-15A (white bars) and -15B (grey bars) splice variants was detected in the same cell lines using specific Taqman quantitative RT-PCR and normalized to TBP. Expression levels are reported as minus mean $\triangle C T$ ( $\triangle C T, C T$ values obtained with TBP primers minus $C T$ values obtained with PFKFB2-15A or -15B primers, respectively). In AT-1, both splice variants, and in NALM6 the PFKFB2-15A variant, could be detected in the absence of dexamethasone, although at very low levels (presumably due to slightly higher efficiency of these RT-PCRs compared to the RT-PCR for both variants). 


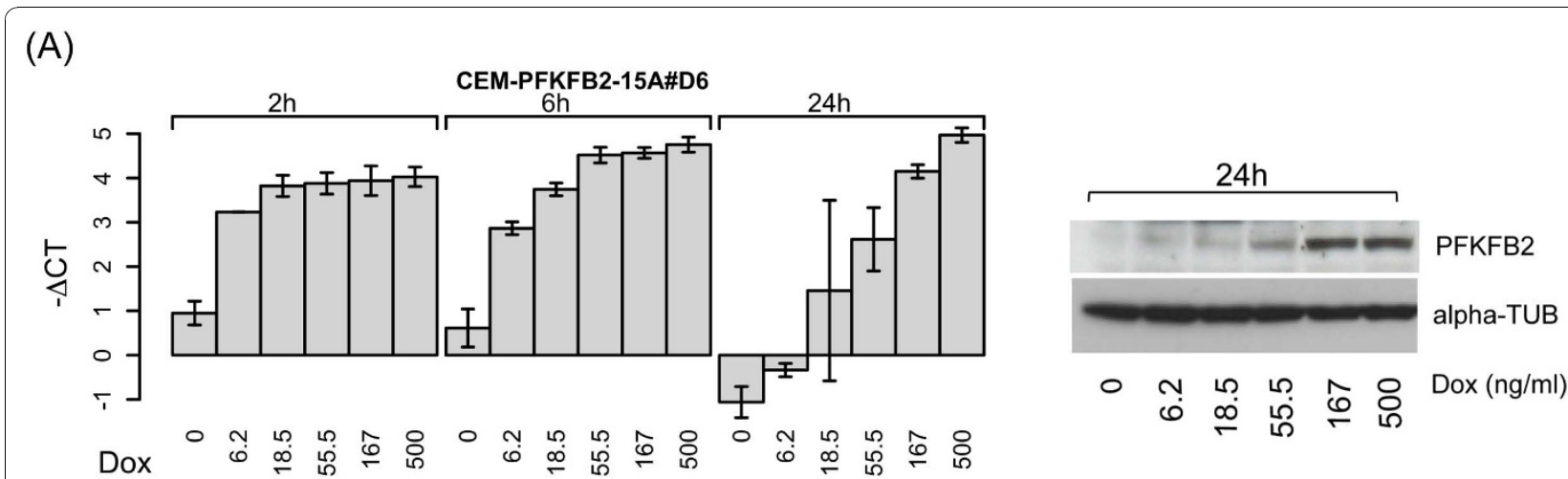

(B)
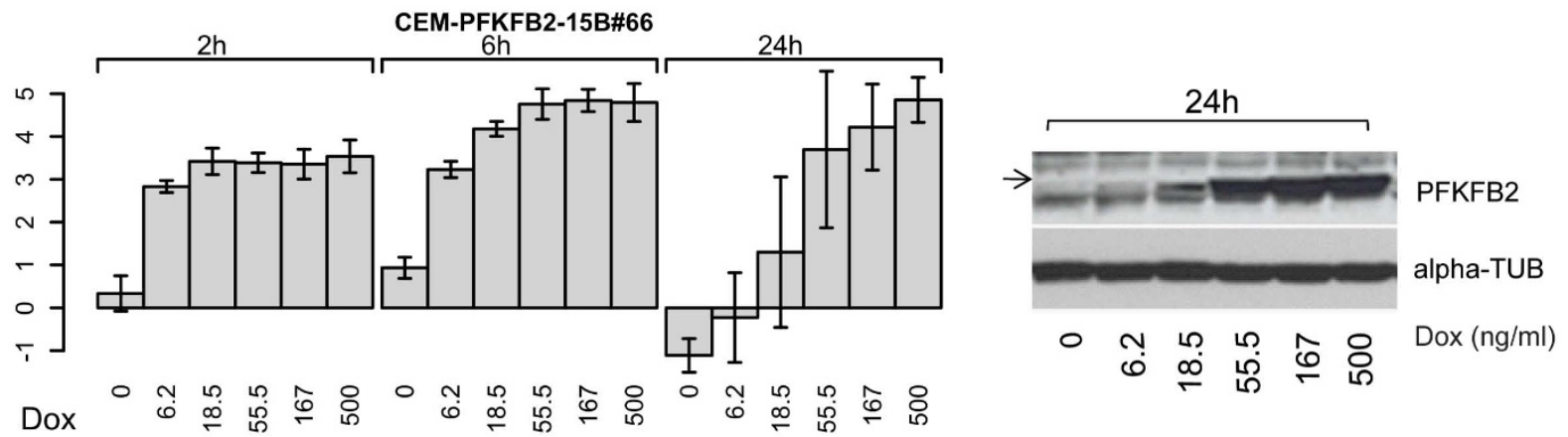

Figure 2 Characterization of T-ALL cell lines with conditional PFKFB2-15A and -15B expression. CEM-PFKFB2-15A \#D6 (Figure 2A) and CEM-PFKFB2-15B \#66 (Figure 2B) cells expressing PFKFB2-15A and -15B in a doxycycline-dependent manner, respectively, were cultured in the presence of increasing concentration of doxycycline (Dox) for the indicated time and analysed for PFKFB2 expression (see Figures S2 and S3 for the corresponding data for the remaining 4 cell lines used in this study). mRNA expression was assessed by using quantitative real time RT-PCR targeting the exons-3/exon-4 boundary present in both splice variants. The data derive from 3 biological replicates analyzed in triplicate and expressed as $-\triangle C T$ (i.e., $C T$ value of PFKFB2-15A minus $C T$ value of TBP). PFKFB2 protein was detected by immunoblotting with antibodies against PFKFB2 and $\alpha$-TUBULIN as loading control (right panels). Shown are representative examples of cells treated with the indicated amounts of doxycycline for 24 hours.

primary malignant lymphoblasts and leukemic cell line models. PFKFB2 was identified by microarray analysis of lymphoblasts isolated from GC-treated children suffering from ALL [5] as one of the most promising candidate genes, since it was regulated in the majority of patients and its deregulation should entail disturbances in glucose metabolism which, in turn, have been implicated in cell death induction in general [12,13]. More specifically, GC resistance was associated with increased glucose consumption [32], and interference with this glucose dependency by simultaneous addition of 2-deoxy-D-glucose (2-DG) increased in vitro sensitivity to GC $[32,33]$. We therefore hypothesized that the apoptotic effect of GC might be triggered, at least in part, by reducing the glycolytic activity of the cell, and that this GC effect is mediated by upregulation of PFKFB2.

To further test this hypothesis, we first performed detailed expression and regulation analysis of the 2 splice variants of this gene and of the 3 other members of the PFKFB gene family in several GC-sensitive and -resistant leukemic T-ALL and preB-ALL cell lines and peripheral blood cells from non-leukemic individuals. This made a possible contribution of the 3 other PFKFB isoenzymes to $\mathrm{GC}$ effects in such cells highly unlikely: PFKFB 1 and -4 were not, or only very moderately, expressed and were not regulated by GC treatment, PFKFB3 was expressed, but not significantly regulated by GC (Additional file 1, Table S3). Concerning $P F K F B 2$, we observed that both splice variants were expressed at similar levels (with PFKFB2-15A somewhat higher than $-15 B$ ) and were co-regulated in most systems. Although the number of patients is limited, GC induction was more pronounced (both in terms of frequency and extent of regulation) in T-ALLs compared to precursor B-ALLs. Concerning correlation with GC sensitivity, all ALL children were considered GC-sensitive because all showed a reduction in blast counts within the first 24 to 48 hours of treatment. Three 


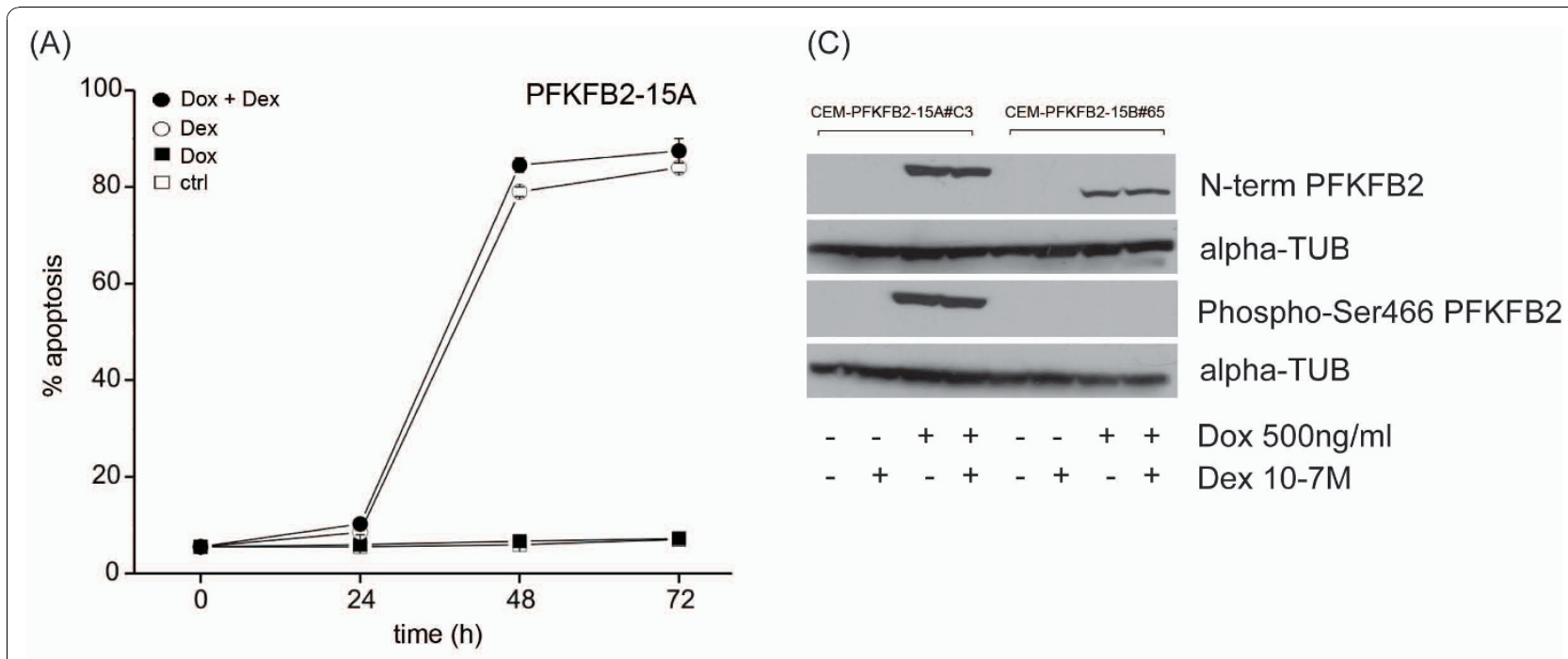

(B)

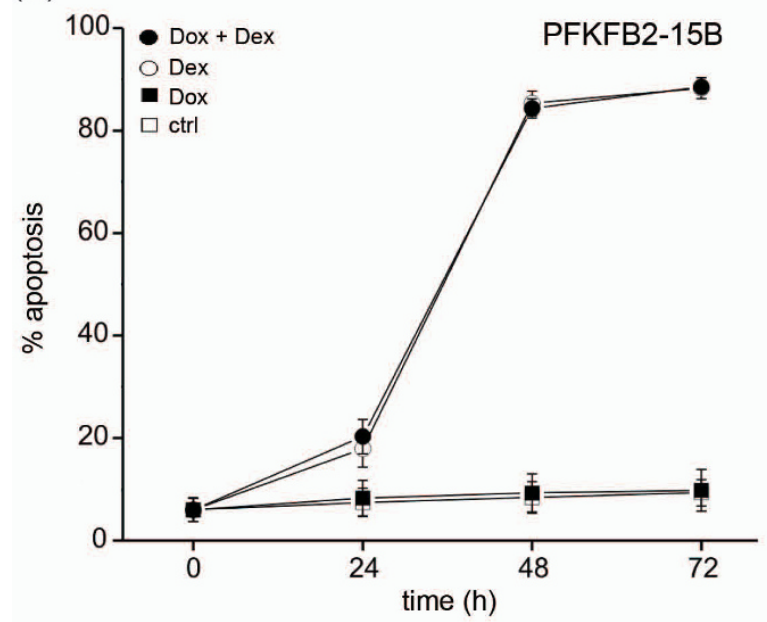

Figure 3 Conditional PFKFB2 over-expression in CCRF-CEM T-ALL cells has no effect on GC-induced apoptosis. CEM-PFKFB2-15A\#C3, \#D6 and \#E8 cells (expressing PFKFB2-15A in a doxycycline-dependent manner, Figure 3A) and CEM-PFKFB2-15A\#65, \#66 and \#95 cells (expressing PFKFB2-15B in a doxycycline-dependent manner, Figure 3B) were cultured in the absence of doxycycline and dexamethasone (open square), in the presence of $10^{-7} \mathrm{M}$ dexamethasone (open circle) or $500 \mathrm{ng} / \mathrm{ml}$ doxycycline (closed square), and with the combination of both (closed circle) for the indicated time and subjected to apoptosis determination using flow cytometric analyses of propidium iodide-stained nuclei ("apoptosis" refers to percent of events in the SubG1 window). Since all 3 cell lines for each isoform gave similar results, the data were pooled. Shown are the mean values $\pm S D$ of specific apoptosis derived from biological triplicates of the 3 cell lines (i.e., 9 measurements per time point and treatment). Figure 3C: PFKFB2-15A and -15B protein expression was analyzed in the indicated cell lines with and without $24 \mathrm{~h}$ doxyxcyline induction (Dox, $500 \mathrm{ng} / \mathrm{ml}$ ) and in the absence and presence of dexamethasone (Dex, $10^{-7} \mathrm{M}$ ) by immunoblotting using an antibody against the shared N-terminal region (top panel) or Ser466 phosphorylated PFKFB2-15A ( $3^{\text {rd }}$ panel) with $\alpha$-TUBULIN serving as loading control.

children $(24,699,724)$ were classified as prednisolone poor responders based on a blast count of $>1000 / \mu \mathrm{l}$ on day 8 , yet they also showed GC-induction of at least 1 of the variants at 1 or both time points. GC-resistant cell lines and non-leukemic individuals (whose peripheral blood lymphocytes are considered resistant to GC-induced apoptosis) [34,35], clearly showed less GCinduction of PFKFB2. Thus, even though the correlation of GC-induction of PFKFB2 and cell death was not complete, the extended expression/regulation analyses further supported a possible role of PFKFB2 in GC-induced apoptosis, particularly in T-ALL cells.

We therefore performed conditional over-expression of both PFKFB2 splice variants in a GC-sensitive T-ALL model system, but these experiments showed no detectable effect of PFKFB2-15A or -15B on cell death, nor did their over-expression change sensitivity to, or kinetics of, GC-induced apoptosis. While these data clearly showed that induction of neither of the 2 splice variants suffices to elicit the anti-leukemic GC effects, 
further work is needed to dissect whether the effects of GC on glucose metabolism are mediated by induction of PFKFB2 (as we hypothesized based on the reported role of PFKFB enzymes) or whether other, perhaps less obvious, pathways are involved. Preliminary experiments addressing this issue suggested that, surprisingly, overexpression of both PFKFB2 splice variants seems to have little, if any, effect on lactate and ATP production, two indicators of glycolytic activity that are reduced after GC treatment. Thus, the current data show that induction of the 2 splice variants of PFKFB2 cannot explain the anti-leukemic GC effects. Whether alterations of glucose metabolism by other GC-dependent mechanisms might contribute to GC-induced leukemia apoptosis requires further investigations.

\section{Conclusions}

Our study demonstrated that the heart-specific PFKFB2 isoenzyme is expressed and specifically induced by GC in malignant lymphoid cells, however, functional analysis of this gene in the human T-ALL cell line model CCRFCEM revealed that its over-expression does not explain the anti-leukemic effects of GC.

\section{Additional material}

Additional file 1: Supplement. Supplementary information consisting of 3 Tables, 4 Figures and additional discussion section.

\begin{abstract}
Acknowledgements
The authors would like to thank Drs. Stephan Geley and A. Trockenbacher for stimulating discussions, M. Brunner, K. Götsch, D. Grazio, B. Gschirr, A. Kofler, S. Lobenwein, C. Mantinger for technical assistance and M. Kat Occhipinti-Bender for editing the manuscript. Supported by the Austrian Science Fund (SFB-F021, P18747), and ONCOTYROL, a COMET Center funded by the Austrian Research Promotion Agency (FFG), the Tiroler Zukunftsstiftung and the Styrian Business Promotion Agency (SFG). The Tyrolean Cancer Research Institute is supported by the "Tiroler Landeskrankenanstalten Ges.m.b.H. (TILAK)", the "Tyrolean Cancer Aid Society", various businesses, financial institutions and the People of Tyrol.
\end{abstract}

\section{Author details \\ ${ }^{1}$ Division Molecular Pathophysiology, Biocenter, Medical University of Innsbruck, Innsbruck, Austria. ${ }^{2}$ Tyrolean Cancer Research Institute, Innsbruck, 6020-Austria. ${ }^{3}$ Department of Hematology and Oncology, Medical University of Innsbruck, Innsbruck, Austria. ${ }^{4}$ Children's Cancer Research Institute and St. Anna Kinderspital, Vienna, Austria. ${ }^{5}$ Department of Pediatrics I, Medical University of Innsbruck, Innsbruck, Austria. 'Department of Pediatrics II, Medical University of Innsbruck, Innsbruck, Austria. Institute of Legal Medicine, Medical University Innsbruck, Innsbruck, Austria.}

\section{Authors' contributions}

MC participated in most experiments and contributed to the writing of the manuscript. KJ generated the PFKFB2 constructs, JR performed the bioinformatic data analyses, SS, RP, GM, MP and BM provided clinical samples and corresponding data, CP coordinated the writing of the manuscript, and RK coordinated the entire study and contributed to the writing of the manuscript. The final version was seen and approved by all authors.

\section{Competing interests}

The authors declare that they have no competing interests.

Received: 8 July 2010 Accepted: 23 November 2010

Published: 23 November 2010

\section{References}

1. Schmidt S, Rainer J, Ploner C, Presul E, Riml S, Kofler R: Glucocorticoidinduced apoptosis and glucocorticoid resistance: Molecular mechanisms and clinical relevance. Cell Death Differ 2004, 11(Suppl 1):S45-S55.

2. Distelhorst CW: Recent insights into the mechanism of glucocorticosteroid-induced apoptosis. Cell Death Differ 2002, 9:6-19.

3. Gross KL, Lu NZ, Cidlowski JA: Molecular mechanisms regulating glucocorticoid sensitivity and resistance. Mol Cell Endocrinol 2009, 300:7-16.

4. Tissing WJ, Meijerink JP, den Boer ML, Pieters R: Molecular determinants of glucocorticoid sensitivity and resistance in acute lymphoblastic leukemia. Leukemia 2003, 17:17-25.

5. Schmidt S, Rainer J, Riml S, Ploner $C$, Jesacher $S$, Achmüller $C$, Presul E, Skvortsov S, Crazzolara R, Fiegl M, Raivio T, Jänne OA, Geley S, Meister B, Kofler R: Identification of glucocorticoid response genes in children with acute lymphoblastic leukemia. Blood 2006, 107:2061-2069.

6. Heine-Suner D, Diaz-Guillen MA, Lange AJ, Rodriguez dC: Sequence and structure of the human 6-phosphofructo-2-kinase/fructose-2,6bisphosphatase heart isoform gene (PFKFB2). Eur J Biochem 1998, 254:103-110.

7. Rider MH, Bertrand L, Vertommen D, Michels PA, Rousseau GG, Hue L: 6-phosphofructo-2-kinase/fructose-2,6-bisphosphatase: head-to-head with a bifunctional enzyme that controls glycolysis. Biochem J 2004, 381:561-579.

8. Colosia AD, Lively M, El-Maghrabi MR, Pilkis SJ: Isolation of a cDNA clone for rat liver 6-phosphofructo 2-kinase/fructose 2,6-bisphosphatase. Biochem Biophys Res Commun 1987, 143:1092-1098.

9. Okar DA, Manzano A, Navarro-Sabate A, Riera L, Bartrons R, Lange AJ: PFK2/FBPase-2: maker and breaker of the essential biofactor fructose-2,6bisphosphate. Trends Biochem Sci 2001, 26:30-35.

10. Kitamura K, Kangawa K, Matsuo H, Uyeda K: Phosphorylation of myocardial fructose-6-phosphate,2-kinase: fructose-2,6-bisphosphatase by CAMP-dependent protein kinase and protein kinase C. Activation by phosphorylation and amino acid sequences of the phosphorylation sites. J Biol Chem 1988, 263:16796-16801.

11. Bertrand L, Alessi DR, Deprez J, Deak M, Viaene E, Rider MH, Hue L: Heart 6-phosphofructo-2-kinase activation by insulin results from Ser-466 and Ser-483 phosphorylation and requires 3-phosphoinositide-dependent kinase-1, but not protein kinase B. J Biol Chem 1999, 274:30927-30933.

12. Plas DR, Rathmell JC, Thompson CB: Homeostatic control of lymphocyte survival: potential origins and implications. Nat Immunol 2002, 3:515-521.

13. Tennant DA, Duran RV, Gottlieb E: Targeting metabolic transformation for cancer therapy. Nat Rev Cancer 2010, 10:267-277.

14. Rathmell JC, Vander Heiden MG, Harris MH, Frauwirth KA, Thompson CB: In the absence of extrinsic signals, nutrient utilization by lymphocytes is insufficient to maintain either cell size or viability. Mol Cell 2000, 6:683-692.

15. Hammerman PS, Fox CJ, Thompson CB: Beginnings of a signaltransduction pathway for bioenergetic control of cell survival. Trends Biochem Sci 2004, 29:586-592.

16. Warburg O: Ist die aerobe Glykolyse spezifisch für die Tumoren? Biochem Z 1929, 204:482-487.

17. Vander Heiden MG, Cantley LC, Thompson CB: Understanding the Warburg effect: the metabolic requirements of cell proliferation. Science 2009, 324:1029-1033.

18. Strasser-Wozak EMC, Hattmannstorfer R, Hála M, Hartmann BL, Fiegl M, Geley S, Kofler R: Splice site mutation in the glucocorticoid receptor gene causes resistance to glucocorticoid-induced apoptosis in a human acute leukemic cell line. Cancer Res 1995, 55:348-353.

19. Schmidt S, Irving JA, Minto L, Matheson E, Nicholson L, Ploner A, Parson W, Kofler A, Amort M, Erdel M, Hall A, Kofler R: Glucocorticoid resistance in two key models of acute lymphoblastic leukemia occurs at the level of the glucocorticoid receptor. FASEB J 2006, 20:2600-2602. 
20. Löffler M, Tonko M, Hartmann BL, Bernhard D, Geley S, Helmberg A, Kofler R: c-myc does not prevent glucocorticoid-induced apoptosis of human leukemic lymphoblasts. Oncogene 1999, 18:4626-4631.

21. Gossen M, Freundlieb S, Bender G, Müller G, Hillen W, Bujard H: Transcriptional activation by tetracyclines in mammalian cells. Science 1995, 268:1766-1769.

22. Helmberg A, Auphan N, Caelles C, Karin M: Glucocorticoid-induced apoptosis of human leukemic cells is caused by the repressive function of the glucocorticoid receptor. EMBO J 1995, 14:452-460.

23. Fears S, Chakrabarti SR, Nucifora G, Rowley JD: Differential expression of TCL1 during pre-B-cell acute lymphoblastic leukemia progression. Cancer Genet Cytogenet 2002, 135:110-119.

24. Parson W, Kirchebner R, Mühlmann R, Renner K, Kofler A, Schmidt S, Kofler $R$ : Cancer cell line identification by short tandem repeat profiling: power and limitations. FASEB J 2005, 19:434-436.

25. Wu Z, Irizarry RA, Gentleman RC, Martinez Murillo F, Spencer F: A model based background adjustment for oligonucleotide expression arrays. Internet 2004 [http://www.bepress.com/jhubiostat/paper1/].

26. Gentleman RC, Carey VJ, Bates DM, Bolstad B, Dettling M, Dudoit S, Ellis B, Gautier L, Ge Y, Gentry J, Hornik K, Hothorn T, Huber W, lacus S, Irizarry R, Leisch F, Li C, Maechler M, Rossini AJ, Sawitzki G, Smith C, Smyth G, Tierney L, Yang JY, Zhang J: Bioconductor: open software development for computational biology and bioinformatics. Genome Biol 2004, 5:R80.

27. Nicoletti I, Migliorati G, Pagliacci MC, Grignani F, Riccardi C: A rapid and simple method for measuring thymocyte apoptosis by propidium iodide staining and flow cytometry. J Immunol Methods 1991, 139:271-279.

28. Geley S, Hartmann BL, Hattmannstorfer R, Löffler M, Ausserlechner MJ, Bernhard D, Sgonc R, Strasser-Wozak EMC, Ebner M, Auer B, Kofler R: P53induced apoptosis in the human T-ALL cell line CCRF-CEM. Oncogene 1997, 15:2429-2437.

29. Gruber $G$, Carlet $M$, Turtscher E, Meister B, Irving JA, Ploner C, Kofler R: Levels of glucocorticoid receptor and its ligand determine sensitivity and kinetics of glucocorticoid-induced leukemia apoptosis. Leukemia 2009, 23:820-823.

30. Ploner C, Rainer J, Niederegger H, Eduardoff M, Villunger A, Geley S, Kofler R: The BCL2 rheostat in glucocorticoid-induced apoptosis of acute lymphoblastic leukemia. Leukemia 2008, 22:370-377.

31. Mansha M, Carlet M, Ploner C, Gruber G, Wasim M, Wiegers GJ, Rainer J, Geley S, Kofler R: Functional analyses of Src-like adaptor (SLA), a glucocorticoid-regulated gene in acute lymphoblastic leukemia. Leuk Res 2010, 34:529-534.

32. Hulleman E, Kazemier KM, Holleman A, Vanderweele DJ, Rudin CM, Broekhuis MJ, Evans WE, Pieters R, den Boer ML: Inhibition of glycolysis modulates prednisolone resistance in acute lymphoblastic leukemia cells. Blood 2009, 113:2014-2021.

33. Eberhart K, Renner K, Ritter I, Kastenberger M, Singer K, Hellerbrand C, Kreutz M, Kofler R, Oefner PJ: Low doses of 2-deoxy-glucose sensitize acute lymphoblastic leukemia cells to glucocorticoid-induced apoptosis. Leukemia 2009, 23:2167-2170.

34. Nieto MA, González A, Gambón F, Díaz-Espada F, López-Rivas A: Apoptosis in human thymocytes after treatment with glucocorticoids. Clin Exp Immunol 1992, 88:341-344.

35. Brunetti M, Martelli N, Colasante A, Piantelli M, Musiani P, Aiello FB: Spontaneous and glucocorticoid-induced apoptosis in human mature T lymphocytes. Blood 1995, 86:4199-4205.

\section{Pre-publication history}

The pre-publication history for this paper can be accessed here: http://www.biomedcentral.com/1471-2407/10/638/prepub

doi:10.1186/1471-2407-10-638

Cite this article as: Carlet et al:. Expression, regulation and function of phosphofructo-kinase/fructose-biphosphatases (PFKFBs) in glucocorticoid-induced apoptosis of acute lymphoblastic leukemia cells. BMC Cancer 2010 10:638.

\section{Submit your next manuscript to BioMed Central and take full advantage of:}

- Convenient online submission

- Thorough peer review

- No space constraints or color figure charges

- Immediate publication on acceptance

- Inclusion in PubMed, CAS, Scopus and Google Scholar

- Research which is freely available for redistribution

Submit your manuscript at www.biomedcentral.com/submit
Biomed Central 